\title{
Ceramic Nanomaterials for High Temperature Applications
}

\author{
Mikhail Gamero* \\ Department of Materials Science \& Engineering, USA \\ *Corresponding author: Mikhail Gamero, Department of Materials Science \& Engineering, Raleigh, NC 27695, USA,
}

Submission: 眥 November 02, 2017; Published: 眥 November 29, 2017

\begin{abstract}
Ceramic nanomaterials have exhibited extraordinary characteristics, based in its low density, hardness and when properly engineered, good corrosion properties in high temperature applications. In the nanoscale world, we can find nanomaterials that do not necessarily apply to the correlation of strength and density. It has been studied that ceramic nanomaterials have some control boundaries that are critical in high temperature applications; grain sizes, grain boundaries density, operating conditions (thermal cycles), nano-crystalline structure and large voids (because of the porous nature of ceramic materials).

In this research paper, it will be discussed some of the most important applications, processing methods and several adhesion methods to metal substrates with the final purpose to enhance metal properties and mitigate high temperature corrosion. As a quick review, it will be discussed some of the environmental health \& safety (EHS) concerns and some parallel experiments that could be done for reinforcing the investigations done in this subject.
\end{abstract}

\section{Ceramic Nanomaterial Applications}

One of the most studied applications is the use of ceramic nanoparticles as pigments to enhance the material properties and products life-cycle. This application is more focused to polymers. The idea is a mitigation of the polymer degradation that can be observed when exposed to ultraviolet light. It has been observed that with the proper blending and binder's ratio, the mechanical and elevated temperature properties can be improved. Ceramic pigments have shown to be a good option in the nano-level for high quality coatings. As reported by Sankar S et al. [1] ceramic pigments show good impact-resistance for ferrous metals and its application in corrosive environments as well as high temperature applications based in the thermal behavior of the coating.

One of the most important applications for this nano-materials, it's the direct application to aggressive environments or high temperature applications like nozzle tips for the inside combustion parts in the automotive industry or its use in turbomachinery, more specific, in combustion and hot gas path components of aviation engines or heavy duty gas turbines for the power business. For being able to apply this technology, it is important to consider the adhesion into the metal substrate of the ceramic coating, where spallation, cracking or not-homogeneous distribution can be observed when improperly added together.

As reported by Vert $\mathrm{R}$ et al. [2] air plasma spraying technique was used for making a thin film of few micrometers thick, where it was confirmed by Vickers indentation and tensile tests, the compatibility of these two different materials (metal substrate with a ceramic coating).

\section{Processing Methods}

There are several processing methods that will be briefly discussed in this section, some of this are in experimental phase as nano-powder infiltrated transient eutectic phase (NITE) process, sol-gel method and carbon nanotubes. As reported by Kuznetsov NT [3], NITE based in the use of silicon carbide nano-powders and its mixture of yttrium and aluminum oxides to be subjected to a temperature of $1800-1950{ }^{\circ} \mathrm{C}$ at a pressure of $15-20 \mathrm{MPA}$ (usually referred as hot pressing) so that the melted film favors the orientation and a good matrix control of the nanoparticles.

With NITE method, good mechanical characteristics are exhibited along with stress limit of 400MPA and an elasticity modulus of 310GPA with good oxidation resistance. Figure 1 shows a cross section of SiC/SiC NITE micrograph, composite material suitable for high temperature applications. It can be clearly distinguished the fiber from the matrix and its interaction.

Sankar et al. [3], was a silicone resin binder developed by high temperature ceramic pigment nanoparticles with a high hardness that can be used under high temperature or aggressive environment applications. The preparation of the ceramic pigments was through 
cobalt and alumina compounds by a thermal decomposition with a limited supply of air or oxygen (calcination) and crushed. After the raw material process was finished, this concentration was applied to a mild steel specimen by hand application and dried, giving a result of a layer coating of $80 \pm 5 \mu$. The results revealed that the system is not degraded up to $600{ }^{\circ} \mathrm{C}$, which makes a suitable polymer-pigment product that can withstand medium range temperature and aggressive environments [4].

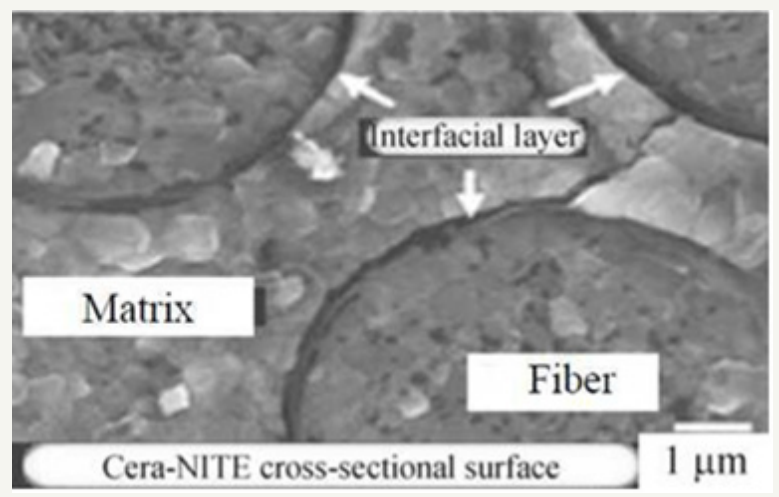

Figure 1: Cross section of NITE $\mathrm{SiC} / \mathrm{SiC}$ [2] other processing method reported by $\mathrm{S}$.

Another elevated temperature application was documented by Chichkan AS et al. [5], which is basically the use of carbon nanomaterials for the creation of ceramic nanomaterials. The preparation of the sample was with the binding of thermochemical activation (TCA) and hydration (OAO Katalizator). A plasticized product was made aqueous using nitric acid which was then dried and compacted to $30 \mathrm{~mm} \times 2 \mathrm{~mm}$ pellets. After obtaining the pellets, it was then subjected to a heat treatment that went from $150{ }^{\circ} \mathrm{C}$ to $1200{ }^{\circ} \mathrm{C}$.

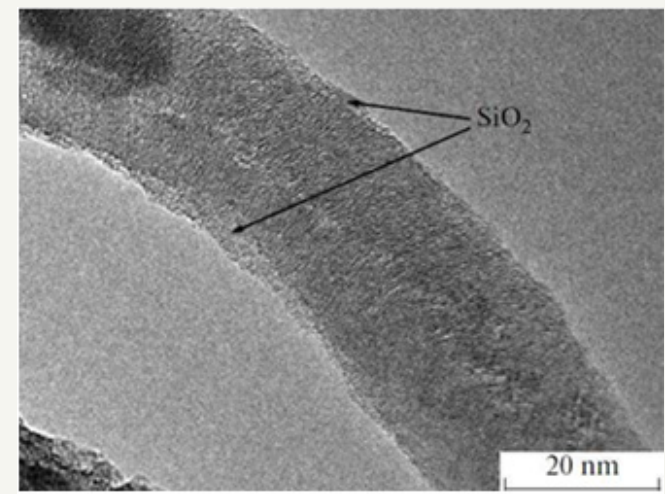

Figure 2: Electron microscopy image of the CNF-SiO composite [1].

The result was a porous structure of $\mathrm{CNM}-\mathrm{SiO}_{2}$ of ceramic membranes that allowed a good control of the porous structure of ceramic membranes, which could be controlled by composites and additives. Figure 2 is an electron microscopy of the carbon nanofilaments $\mathrm{CNF}-\mathrm{SiO}_{2}$ composite where it can be appreciated the $\mathrm{SiO}_{2}$ film produced on the CNM surface improving the ceramic matrix with the formation of mullite phase.

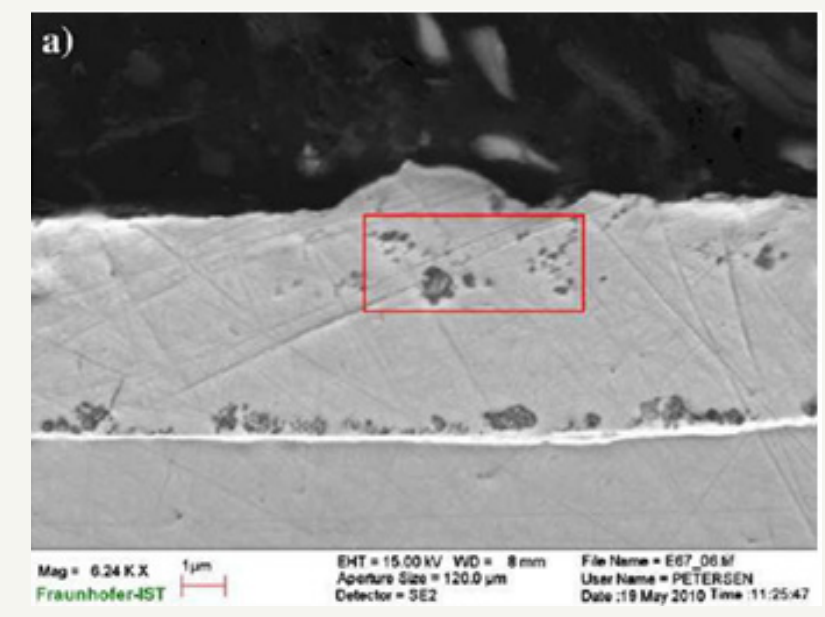

Figure 3a: $\mathrm{TiO}_{2}-\mathrm{Nc}$ concentration $1 \mathrm{~g} / 1$ [6].

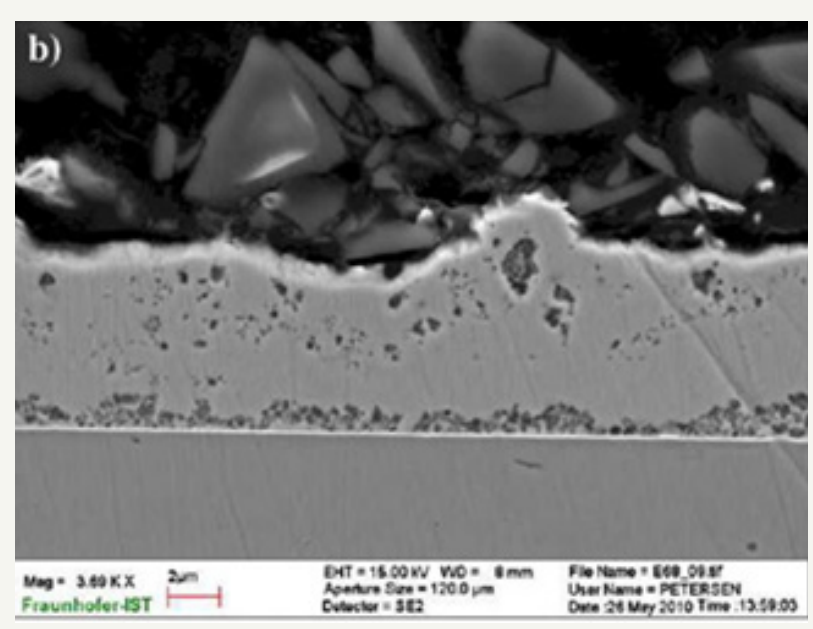

Figure 3b: $\mathrm{TiO}_{2}-\mathrm{Nc}$ concentration $3 g / 1$ [6].

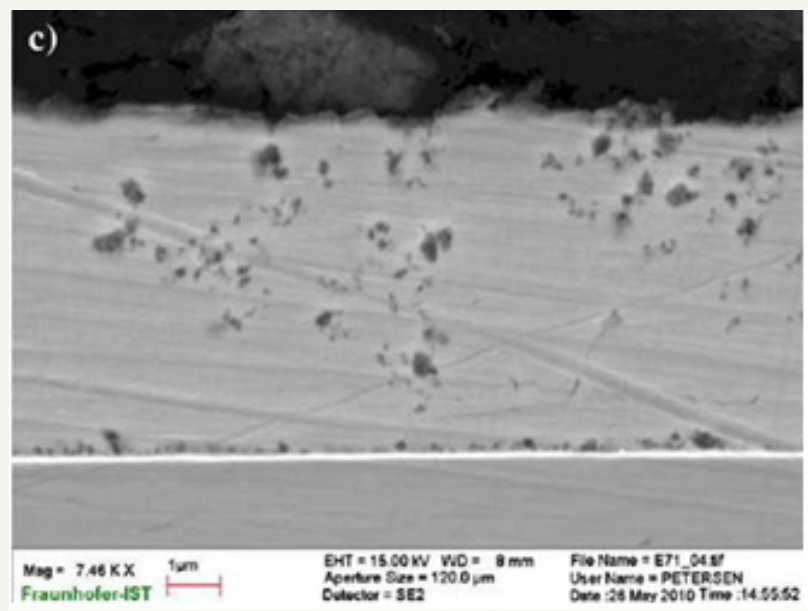

Figure 3c: Coating layer fabricated in two steps [6].

An excellent processing method for nickel composite coating was studied by Moustafa EM et al. [6], where it was discussed the formation of a composite Ni-NC (nanocontainers) for a bi-functional coating. The idea is based in three fundamental steps, adsorption 
of positively charged $\mathrm{TiO}_{2}$-NC on SAM-covered gold coated steel substrate. Secondly, a metalized NC layer by electroplating of a thin $\mathrm{Ni}$ layer and finally, the conduction of current density in the Ni-NC composite for showing good adhesion to the substrate. The result showed an NC finely dispersed in the Ni coating with a high density incorporated which increased the concentration in the Ni solution. It was very interesting how the use of gold coated steel electrodes helped in the stabilization of the SAM showing good adhesion to the metal substrate. A comparison of Figure $3 a, 3 b$ and $3 c$ is for showing the interface between $\mathrm{Au}$ and Ni (Figure 3a), at different $\mathrm{TiO}_{2}$ concentrations (Figure $3 \mathrm{~b}$ ) and in the fabrication with different steps (Figure 3c). The first step was the adsorption of $\mathrm{TiO}_{2}-\mathrm{NC}$ and second, the electroplating for obtaining a $6 \mu \mathrm{m}$ thickness layer.

The last processing method that will be discussed is a ceramic nanomaterial based in a Ti-Si-C-N by sol-gel which was investigated by Biedunkiewicz A [7]. The process is based in two different stages, the first one at low temperature, where the resulting product is raw nc-TiCx material and the second one is at high temperature stage carbonization where the carbon traces (carbides essentially) are eliminated of excessive organic compounds.

The second condition enables the purification and limits the growth of crystallites to ensure the carbonization. After dry-out, the sol-gel solution was evaluated, showing uniform distribution, grains of silicon, titanium and carbon were regularly distributed. The only problem was that a higher oxidation resistance could was not favorable. Figure 4 shows a distribution of Ti-Si-C homogenous distribution.

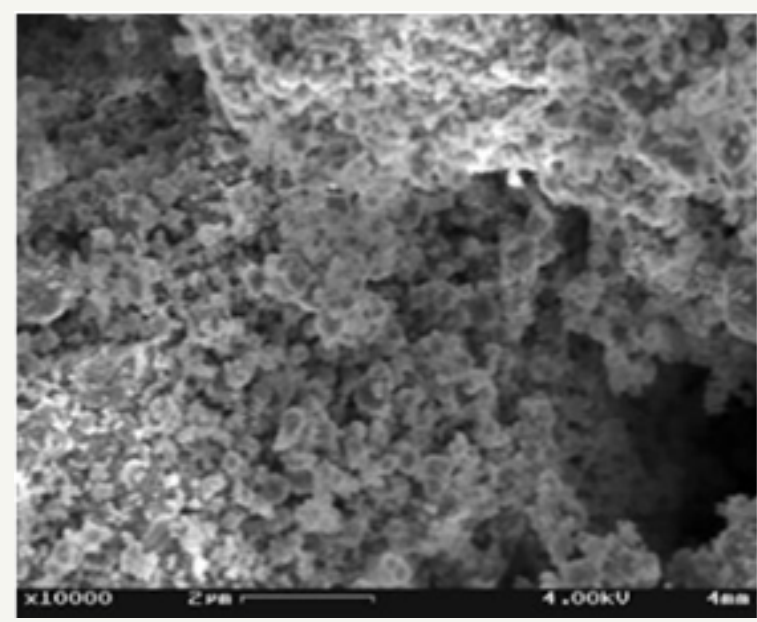

Figure 4: HRSEM image of Ti-Si-C powder [8].

One critical step for the coating use is its adherence to a metal substrate. In his work, Vert R et al. [2], it was studied the adhesion of a coating with a plasma process application. The surface was firstly prepared with a blasting process to make the smooth surface tougher; the blasting process impacts the surface leaving it under a compressive stress. The most suitable application of the coating layer was in two steps, the first one using air plasma spraying (APS) technique and the second one using suspension plasma spraying (SPS), which is essentially spraying a ceramic powder into the surface.
The adhesion of the coating was measured with a hardness test and by a tensile test. It was noticed that after the air plasma spraying (APS) application, if the coating layer was bigger than required, the adhesion was not optimal. Yttria-stabilized Zirconia was sprayed in the already cleaned surface, during the application of the spraying. One critical step for the proper adhesion of the ceramic coating to the metal substrate is a controlled temperature of $200{ }^{\circ} \mathrm{C}$, used in a preheated surface of $400^{\circ} \mathrm{C}$.

The result showed two different surface that are clearly observed with the microscope, the first layer was thin and dense, composed of oxides while the second layer showed a columnar grain structure with a granular structure. Figure 5a shows the microstructure and the difference between the two-applied coating processes. Figure $5 \mathrm{~b}$ shoes the interface of the SPS and APS layers.

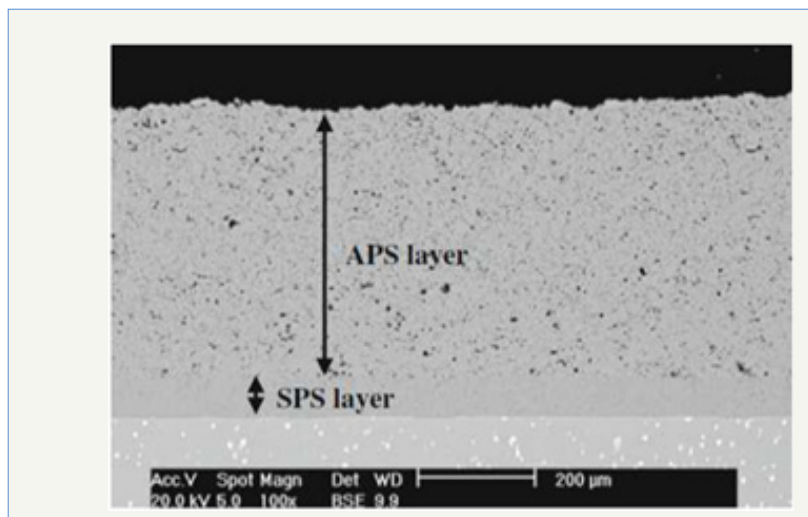

Figure 5a: YSZ microstructure duplex-coat [2].

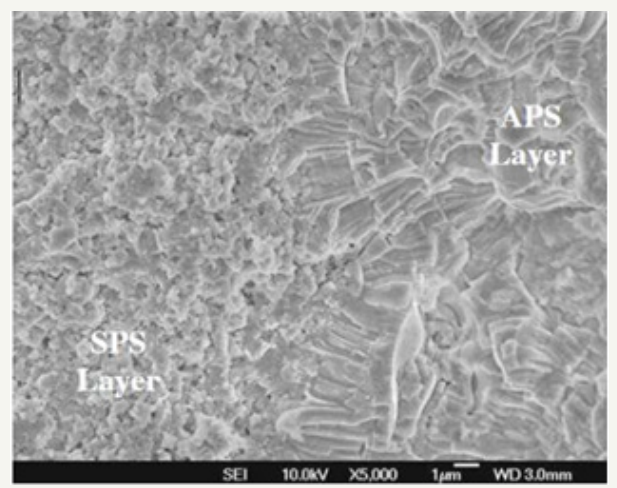

Figure 5b: Interface between SPS and APS layers [2].

The results for the tensile bond strength were between 12-24 $\mathrm{MPa}$, depending on the thickness of each individual layer and the thickness of them both. Vickers indentation test confirmed that the failure of the duplex coating occurrs in the APS coating, closer to SPS-APS interface. The remark of this methodology showed an excellent adhesion of the insulating coating to the metal surface. In other words, the first coating serves as a bond coating between the metal substrate and the thicker coating layer. The coating of the second layer is important because the thicker the layer, the colder the metal substrate surface but the it is also hotter the ceramic coating surface. It is important to find the proper balance because a hotter ceramic surface impacts the life of the part since it is suitable to spallation and cracking. 


\section{Environmental, Health and Safety concerns}

The use of nanomaterials has increased enormously during the last years. Although we are not discussing a biological or disease treatment application in this research, it is important to consider that the EHS concerns increase with a large use but it has not been completely studied.

The use of crystalline silica (like the one used as a matrix for several of the examples of ceramic composites) can raise the question of medical implications. As reported by Johnson SM [8], it has been studied the concern of toxicity for the exposure of this materials and it has pointed in two critical directions, inhalation studies show pulmonary and cardiovascular symptoms which arise the question of its implications for long-term exposition use.

The oxides can cause inflammation because of the accumulation and engineered nanoparticles can cause the blood pressure to become unstable, impacting the myocardial infarction or stroke. Before the use of this nanomaterials at a big scale, it shall be further investigated the EHS implications, and its awareness to engineers, manufacturers and consumers.

\section{Proposal}

TaC-SiC ultrahigh temperature composite was analyzed by Yan $\mathrm{Lu}$ et al. [9]. The main goal was the use of cross-linking nanocomposites prepared by the blend of the precursors in an inert atmosphere (Argon). The precursor was obtained by the blending of poly-carbosilane (PCS) and poly-tantaloxane (PT) for obtaining a metal-ligand interaction for the polymer.

After annealing of $1000-1800{ }^{\circ} \mathrm{C}$, it was obtained a homogeneously distributed sample with traces of free carbon. The annealing precursors at $1600{ }^{\circ} \mathrm{C}$ gave a ceramic nanocomposite with promising application of high temperature and aggressive environment conditions [10-12].

This same ceramic composite TaC-SiC synthesis can be applied to some other compositions consisting of a group IV transition metals, in other words, the use of nitrides, or borides can be used for ultrahigh temperature ceramics as it was TaC-SiC [13].

A finely dispersed network of borides or nitrides could be use along a silica-based matrix. This could be verified with some other experiments using $\mathrm{SiC}$ and $\mathrm{Si}_{3} \mathrm{~N}_{4}$ as the matrix former complimented with nitrides, carbides of borides for the ceramic composite high temperature material [14-16].

\section{Conclusion}

We have discussed several technologies that have been developed for enhancing the high temperature properties of a metal substrate, and more specifically, improving the oxidation resistance. It was further reviewed techniques like NITE and APS/ SPS multilayer coatings, the use of ceramic pigments and more importantly, the adhesion of the ceramic coating to the metal surface and some of the most critical elements to consider during the process (like coating thickness and surface preparation).
Some other critical points discussed were the size of grains and the importance of maintaining the proper conditions like voltage (for Au electrode methodology) and the room temperature during spray nozzle technique process. Additionally, it was discussed several tests of how to characterize the product, like Vickers indentation, tensile tests, coating thickness and to determine if the crack or spallation observed might be related with the procedure followed.

It was discussed how this nanomaterial technologies need further investigation to better understand the problems related to environment, health and safety because of the nature of their scale size and exposure.

There is no question that ceramic coatings are an excellent option for enhancing the high temperature properties and are a potential solution for the corrosion mitigation, which is observed at those aggressive operating conditions. They have been used in combustion elements, hot gas path components (like buckets or nozzles) and even for tip nozzle inside automotive engines $[17,18]$.

Producing homogenous, uniform, dense and crack-free coatings is suitable only with well-controlled manufacturing process. Nanomaterials open an enormous number of options when we talk about different oxides or multilayer coatings.

\section{References}

1. Sankar S, Kalaignan GP, Subramanian S, Amutha K, Marikkannu KR, et al. (2006) Innovative nanoparticle clear coat by ipn-ceramic pigments, synthesis and reactivity in inorganic. Metal-Organic and Nano-Metal Chemistry 36(2): 197-201.

2. Vert R, Carles P, Laborde E, Mariaux G, Meillot E, et al. (2012) Adhesion of ceramic coating on thin and smooth metal substrate: a novel approach with a nanostructured ceramic interlayer. Journal of Thermal Spray Technology 21(6): 1128-1134.

3. Kuznetsov NT, Svast'yanov VG, Simonenko P (2010) Finely dispersed refractory compunds for high-temperature ceramic matrix composite applications. Russian Journal of General Chemistry 80(3): 658-665.

4. Greer J (2014) Ceramic nanomaterials that are light, strong and spongy. News \& analysis materials news, 39.

5. Chichkan' AS, Chesnokov VV, Gerasimov EY, Parmon VN (2013) Production of nanoporous ceramic membranes using carbon nanomaterials. Doklady Physical Chemistry 450(2): 135-137.

6. Moustafa EM, Dietz A, Hochsattel T (2013) Manufacturing of nickel/ nanocontainer composite coatings. Surface \& Coatings Technology 216: 93-99.

7. Biedunkiewicz A (2011) Manufacturing of ceramic nanomaterials in Ti-Si-C-N system by sol-gel method. Journal of Sol-Gel Science and Technology 59(3): 448-455.

8. Johnson SM (2013) Nanoscale ceramics: environmental, health, and safety (EHS) implications. Applied Ceramic Technology 10(4): 577-583.

9. Lu Y, Chen F, An P, Ye L, Qiu W, et al. (2016) Polymer precursor synthesis of TaC-SiC ultrahigh temperature ceramic nanocomposites. The Royal Society of Chemistry 91.

10. Gao W, Li Z, He Y (2012) High temperature oxidation protection using nanocrystalline coatings, The University of Auckland, New Zeland and University of Science and Technology, Woodhead Publishing Limited, Beijing, China. 
11. Zheludkevich ML, Tedim J, Ferreira MGS (2012) 'Smart" coatings for active corrosion protection based on multi-functional micro and nanocontainers. Electrochimica Acta 82: 314-323.

12. Pivinskii YE, Grishpun EM, Gorokhovskii AM (2015) Engineering, manufacturing, and servicing of shaped and unshaped refractories based on highly concentrated ceramic binding $\mathrm{s}$ u s p e n s i o n s. Refractories and Industrial Ceramics 56(3): 245-263.

13. Tonello K, Trombini V, Bressiani AA, Bressiani JC (2012) Ceramic processing of nbc nanometric powders obtained by high energy milling and by reactive milling. Materials Science Forum, Trans Tech Publications, Switzerland.

14. Dey S, Drazin JW, Wang Y, Valdez JA, Holesinger TG, et al. (2015) Radiation tolerance of nanocrystalline ceramics: insights from yttria stabilized zirconia. Scientific Reports, doi:10.1038/srep07746.
15. D’Amato R, Falconieri M, Gagliardi S, Popovici E, Serra E, et al. (2013) Synthesis of ceramic nanoparticles by laser pyrolysis: From research to applications. Journal of Analytical and Applied Pyrolysis 104: 461-469.

16. Fonseca AS, Maragkidou A, Viana M, Querol X, Hämeri K, et al. (2016) Process-generated nanoparticles from ceramic tile sintering: Emissions, exposure and environmental release. Science of the Total Environment 565: 922-932.

17. Castro RHR (2013) On the thermodynamic stability of nanocrystalline ceramics. Materials letters 96: 45-56.

18. Muktepavela F, Grigorjeva L, Kundzins K, Gorokhova E, Rodnyi P (2015) Structure, nanohardness and photoluminescence of $\mathrm{ZnO}$ ceramicsbased on nanopowders. Royal Swedish Academy of Sciences 90(9). 